\title{
LA ICTIOFAUNA FLUVIAL ESPAÑOLA AUTÓCTONA Y SU ESTADO DE CONSERVACIÓN
}

\author{
Casildo FERRERAS CHASCO \\ Departamento de Análisis Geográfico Regional y Geografía Física - Univer- \\ sidad Complutense de Madrid
}

\author{
Recibido: 20/04/2011 \\ Devuelto: 07/08/2011 \\ Aceptado: 05/09/2011
}

\begin{abstract}
RESUMEN: Se analizan los avances en el conocimiento de los peces fluviales españoles autóctonos y su estado de conservación según el Atlas y Libro Rojo de 2001 y publicaciones posteriores y se comparan con los datos de obras anteriores como La lista Roja de 1986 y el Libro Rojo de 1992. Este análisis muestra un claro aumento del número de especies reconocido en nuestro país y un aumento de las especies amenazadas, debido en parte a la descripción de nuevas especies. En el último cuarto de siglo ha pasado de 32 a 50 y el número de especies consideradas endémicas de 16 a 29. Respecto al estado de conservación se observa un claro empeoramiento, pues el número de especies gravemente amenazadas ha experimentado un notable aumento y el de las especies no amenazadas ha pasado de ser mayoría, el $56 \%$, a ser una exigua minoría, el $14 \%$. A ello puede haber contribuido los nuevos criterios de la UICN utilizados actualmente, más exigentes que los anteriores, pero es indudable que la situación sigue empeorando porque los factores con influencia negativa en nuestra ictiofauna siguen actuando.
\end{abstract}

PALABRAS CLAVE: peces fluviales, endemismos, conservación fauna, especies amenazadas, España.

\section{THE CONSERVATION STATUS OF AUTOCHTHONOUS SPANISH RIVER ICHTHYOFAUNA}

ABSTRACT: Progress in the knowledge of the aboriginal Spanish river fishes and their conservation according to the Atlas and Libro Rojo of 2001 and later publications is analyzed and compared with data from earlier works such as the 1986 Lista Roja and the Libro Rojo from 1992. This analysis shows a significant increase in the number of listed species in our country and an additional increase of the number of the endangered species, partially because several new species have been lately described. The number of aboriginal species has grown from 32 to 50 and the number of endemic species from 16 to 29 . Regarding their conservation status a clear worsening is observed because the number of severely threatened species has experienced a significant increase, whereas the number of non-endangered species has been dramatically reduced from 56 to 14 per cent. The new IUCN criteria used today, more demanding than the former ones, may have contributed to this change, but there is no doubt that the situation is going worse because the negative factors that affect the river fish stock in Spain keep on working.

KEY WORDS: fluvial fishes, endemism, fauna conservation, endangered species, Spain. 


\section{INTRODUCCIÓN Y METODOLOGÍA}

Los peces fluviales son posiblemente el componente de nuestra fauna más amenazado y que ha tenido que soportar mayores agresiones y al mismo tiempo el que menos interés despierta entre público y el más olvidado por los geógrafos (FERRERAS y REDONDO, 2004; FERRERAS et AL., 2006; FERRERAS, 2010) por lo que a su conservación se refiere.

Sin embargo, su valor científico y como integrante de nuestra biodiversidad es muy notable, ya que, entre otros rasgos, presenta un alto grado de endemicidad, cuya importancia e interés ha ido aumentando a medida que se avanzaba en su conocimiento. Por ello resulta adecuado analizar la situación actual del conocimiento de nuestra ictiofauna autóctona y la importancia en ella de los endemismos, su estado de conservación y las variaciones en todos estos aspectos en las distintas cuencas fluviales. Por ello, aunque ya hemos destacado algunos de estos aspectos en publicaciones anteriores (FERRERAS y REDONDO, 2004; FERRERAS, 2010), la importancia de los avances conseguidos en los últimos años en el conocimiento de nuestros peces fluviales hace conveniente una revisión y puesta al día de conjunto.

Para ello se han tomado como obras base los sucesivos libros rojos que han ido apareciendo en los últimos decenios, complementados, para los años que van del presente siglo, con el rastreo de novedades en las principales revistas especializadas y fuentes informáticas, entre ellas la Enciclopedia Virtual de los Vertebrados Españoles del Museo Nacional de Ciencias Naturales, que por ahora incluye sólo la lista de referencia y textos de unas pocas especies.

En el primer aspecto, el conocimiento de la riqueza de nuestra ictiofauna, la Lista Roja de 1986 y el Libro Rojo de 1992 de los Vertebrados Españoles reflejan los esfuerzos realizados en los últimos decenios del siglo pasado por rellenar las importantes lagunas en el conocimiento de nuestra fauna y en el caso que nos ocupa de nuestra ictiofauna. La primera síntesis, la lista Roja de 1986, contribuirá a hacer tomar conciencia de las importantes deficiencias que existen en el conocimiento de nuestros peces y de que es necesario un esfuerzo investigador importante. El Libro Rojo de 1992 reflejará los primeros resultados de este esfuerzo investigador que se continuará hasta la actualidad, ya que el número de especies de peces reconocido en nuestros ríos pasa de 32 en 1986 a 42 en 1992, por la descripción de nuevas especies y el reconocimiento a nivel específico de taxones descritos anteriormente. El Atlas y Libro Rojo de nuestros peces de 2001 aumenta la riqueza de nuestra ictiofauna hasta 45 especies y señala en varias especies que son necesarias nuevas investigaciones que probablemente darán como resultado la descripción de nuevas especies. En años posteriores se confirmará lo acertado de estas 
apreciaciones, ya que el número de especies de peces actualmente reconocidas en nuestros ríos se eleva a 55 .

En el plano conservacionista, los criterios utilizados en la Lista Roja (1986) y el Libro Rojo (1992) son los mismos, pero distintos de los actuales, que son los de la UICN (Unión Internacional para la Conservación de la Naturaleza) aceptados en el Atlas de 2001. Esto plantea algunos problemas, que se irán tratando posteriormente en el momento adecuado en cada caso, en la interpretación de las variaciones en el estado de conservación de determinadas especies entre 1992 y 2001, ya que puede darse el caso, como en la lamprea de arroyo, de que un cambio de la denominación del estado de conservación pueda reflejar un cambio de criterio y no un deterioro o mejora en su situación real.

Para el conocimiento de los aspectos territoriales, los mapas del Atlas y las bases de datos del MARM (Ministerio de Medio Ambiente, Rural y Marino) son la base fundamental tenida en cuenta, y, para las especies descritas posteriormente, las indicaciones de los autores de su publicación.

\section{RIQUEZA DE NUESTRA ICTIOFAUNA E IMPORTANCIA DE LOS ENDEMISMOS}

Los avances realizados en los últimos decenios, a los que hemos aludido, en el conocimiento de la ictiofauna fluvial española han permitido destacar mejor su valor y significado biológico y evolutivo. Este mejor conocimiento se ha traducido en la descripción de nuevas especies y subespecies ibéricas, en buena parte endémicas, y en la elevación al rango específico de taxones tratados anteriormente a nivel infraespecífico.

El cuadro adjunto refleja claramente estos rasgos básicos pues el número de especies reconocidas ha aumentado de 32, en la Lista Roja de 1986, a 50 en la actualidad, y el de endemismos de 14 a 29. Estos cambios han afectado principalmente al orden cipriniformes y muy especialmente a la familia ciprínidos que ha pasado a representar el $60 \%$ de nuestra ictiofauna y el $83 \%$ de los endemismos. Conviene sin embargo señalar brevemente algunos rasgos o cambios de interés en el resto de los órdenes y familias.

Los primeros órdenes concentran las especies migratorias de nuestros peces continentales. Los anguiliformes presentan la única especie migradora catadroma de nuestra ictiofauna, la anguila (Anguilla anguilla) y los representantes de los órdenes, petromizontiformes, acipenseriformes, clupeiformes y salmoniformes son migradores anádromos, con excepción de la lamprea de arroyo (Lampretra planeri) y la trucha común (Salmo truta). El cambio de número de especies en los petromyzontiformes es más aparente 
que real, pues se debe a que la lamprea de río (Lampetra fluviatilis) no figura en la Lista roja de 1986. Esta lamprea está extinguida en nuestro país, la última cita data de 1974 en el tramo toledano del río Guadarrama, pero sobrevive en Portugal y el mantenerla en los libros y listados posteriores tal vez pueda contribuir a que se tomen medidas que faciliten un regreso que hoy por hoy es improbable.

Cuadro 1. Número de especies autóctonas y de endemismos por familias en 1986 y en la actualidad.

\begin{tabular}{|c|c|c|c|c|c|}
\hline \multirow[b]{2}{*}{ Orden } & \multirow[b]{2}{*}{ Familia } & \multicolumn{2}{|c|}{$n^{o}$ especies } & \multicolumn{2}{|c|}{$n^{o}$ endemismos } \\
\hline & & 1986 & 2011 & 1986 & 2011 \\
\hline Petromyzontiformes & Petromyzontidae & 2 & 3 & & \\
\hline Acipenseriformes & Acipenseridae & 1 & 1 & & \\
\hline Clupeiformes & Clupeidae & 2 & 2 & & \\
\hline Anguilliformes & Anguillidae & 1 & 1 & & \\
\hline Salmoniformes & Salmonidae & 2 & 2 & & \\
\hline \multirow[t]{3}{*}{ Cypriniformes } & Cyprinidae & 16 & 30 & 11 & 24 \\
\hline & Cobitidae & 2 & 3 & 2 & 3 \\
\hline & Balitoridae & 1 & 1 & & \\
\hline \multirow[t]{2}{*}{ Cyprinodontiformes } & Cyprinodontidae & 1 & 1 & & 1 \\
\hline & Valenciidae & 1 & 1 & 1 & 1 \\
\hline Atheriniformes & Atherinidae & 1 & 1 & & \\
\hline Gasterosteiformes & Gasterosteidae & 1 & 1 & & \\
\hline Scorpaeniformes & Cottidae & & 1 & & \\
\hline Perciformes & Blenniidae & 1 & 1 & & \\
\hline Syngnathiformes & Syngnathidae & & 1 & & \\
\hline Total & 15 & 32 & 50 & 14 & 29 \\
\hline
\end{tabular}

Los cambios acaecidos en la taxonomía de la familia de los ciprínidos, la más diversificada de nuestra ictiofauna fluvial, han sido profundos y no se han limitado a la descripción de nuevas especies sino que se han producido también a nivel de género. En una primera etapa se fue produciendo la sinonimización de géneros como Rutilus, Leucissus, Iberocypris y Tropidophoxinellus cuyas especies pasan a ser incluidas en los géneros Chondrostoma y Squalius. Posteriormente se ha producido el proceso contrario y ha aumentado el número de géneros al dividirse el género Barbus en dos, Barbus y $\mathrm{Lu}$ ciobarbus, y el género Chondrostoma en cuatro, Achondrostoma, Iberochondrostoma, Parachondrosotma y Pseudochondrostoma. En los dos primeros se encuadran las especies anteriormente incluidas en Tropidophoxinellus y Leuciscus y en los últimos las englobadas en los grupos Chondrostoma toxostoma y Chondrostoma polylepis respectivamente. 
Por todo ello los cambios han afectado de modo profundo al conjunto de la familia y concretamente a sus tres géneros más importantes Barbus, Chondrostoma, y Squalius y merecen ser tratados con cierto detenimiento.

Por lo que se refiere al género Barbus la Lista Roja (ICONA, 1986) solo reconoce cinco especies pues los barbos de Graells (Barbus graellsii), mediterráneo (B. guiraonis) y gitano (B. sclateri) son consideradas subespecies del barbo común (B. bocagei). Los tres figuran ya como especies independientes en Libro Rojo de 1992, con lo se elevan a ocho las especies de barbos ibéricos, cifra que se mantiene actualmente y de las que seis serían endémicas.

Posteriormente tendrá lugar la división en dos géneros, para cuya validez definitiva en este y otros casos habrá que esperar. Luciobarbus englobará las especies de los grandes ríos meseteños: el barbo común (Luciobarbus bocagei) de las cuencas del Duero y Tajo, el barbo comizo (Luciobarbus comizo) de las del Tajo y Guadiana y el barbo cabecicorto (Luciobarbus microcephalus) exclusivo del Guadiana.

Los otros cinco barbos de las regiones del sur y del este peninsular se mantienen dentro del género Barbus. Tres son endemismos: el barbo gitano (Barbus sclateri) de las regiones del sur, el barbo mediterráneo (Barbus guaironis) del levante peninsular y el colirrojo (Barbus haasi) de la cuenca del Ebro. Los dos no endémicos son el barbo de Graells (Barbus graellsi), también de la cuenca del Ebro, y el barbo de montaña (Barbus meridionalis) del norte de Cataluña.

Respecto al género Chondrostoma en la citada Lista Roja de 1986 sólo figuraban dos especies: Ch. toxostoma para la vertiente mediterránea y $C h$. polylepis para la atlántica. Ambas fueron divididas (1992) en tres especies ibéricas cada una: el grupo toxostoma formado por la loina (Ch. arrigionis), la madrilla (Ch. miegii) y la madrija (Ch. turiense), y el grupo polylepis que incluirá la boga del Duero (Ch. duriense), la boga de río o del Tajo ( $C h$. polylepis s.str.) y la boga del Guadiana (Ch. willkommii).

A este género se asignaron por otro lado la bermejuela (Ch. arcasii) y la pardilla (Ch. lemmingii) anteriormente incluidos en el género Rutilus, con lo que el género Chondrostoma, incluía según el Atlas y Libro Rojo (2001) ocho especies todas ellas endémicas de la península ibérica.

El número de especies españolas del complejo Chondrostoma aumentará hasta diez por la asignación a dos de sus nuevos géneros de sendas nuevas especies también endémicas que se describirán recientemente: la bogardilla (Iberochondrostoma oretanum) y la sarda (Achondrostoma salmantinum). 
La distribución taxonómica y territorial del complejo Chondrostoma y sus nuevos géneros va a quedar del siguiente modo:

Al género Achondrostoma se asignarán dos especies, la bermejuela (A. arcasii), ampliamente distribuida por la mitad norte peninsular del bajo Júcar y el Tajo medio hacia el norte, y la sarda (A. salmantinum).

Dos especies integrarán también el género Iberochondrostoma: la pardilla (I. lemnigii), distribuida del Tajo al bajo Guadalquivir, y la pardilla oretana (I. oretanum) endemismo de la cuenca del Jándula.

En el género Parachondrostoma se van a incluir las tres subespecies anteriormente incluidas en el Chondrostoma toxostoma: la loina ( $P$. arrigionis), de la cuenca del Júcar, la madrija ( $P$. turiense) de la del Turia y la madrilla (P. miegii) de las del Ebro y Cantábrico oriental.

Por último las tres restantes, tradicionalmente llamadas boga de río y que pueblan los ríos atlánticos, que se incluían en Chondrostoma polylepis, pasan a integrar el género Pseudochondrostoma: la boga del Duero (Ps. duriense), la boga del Tajo (Ps. polylepis) en la cuenca de este río y con translocaciones en las del Júcar y Segura, y la del Guadiana ( $P$ s. willkommiii) que además del río que la da nombre coloniza también la cuenca del Guadalquivir y la parte occidental de la vertiente mediterránea andaluza.

El género Squalius, el tercero en importancia de la familia ciprínidos, ha sufrido también cambios importantes. Por un lado incluye hoy especies inicialmente ubicadas en otros géneros (Leuciscus, Tropidophoxinellus e Iberocypris) y por otro su número se ha incrementado con descripciones recientes. Las especies reconocidas más antiguas son: el calandino (Squalius alburnoides (=Tropidophoxinellus alburnoides)), el bagre (S. cepahlus (=Leuciscus cephalus)) y el cacho (S. pyrenaicus (=Leuciscus pyrenaicus)). En 1980 se describe la bogardilla y en 1987 el bordallo (S. caroliterti (=Leuciscus carolitertii)).

Posteriormente se añadirán tres nuevas especies de este género, lo cual había sido ya anticipado cuando a propósito del cacho se señala en el reciente Atlas y Libro Rojo la complejidad interna de sus poblaciones "algunas de las cuales deben considerarse especies diferentes" (DOADRIO, 2001).

Estas nuevas especies serán el cacho del Gallo (S. castellanus), el cachuelo malagueño (S. malacitanus) y el cacho levantino (S.valentinus). Con ello se eleva a ocho el número de especies del género, todas endémicas a excepción del bagre. 
En algunos de los cuatro géneros restantes de la familia ciprínidos también se han producido algunos cambios de interés. Así el gobio español, tradicionalmente incluido en Gobio gobio, ha sido segregado del europeo como nueva especie, Gobio lozanoi, que al estar presente en el sur de Francia no puede calificarse de endemismo, aunque para casos se debería proponer el término subendemismo. Algo parecido sucede en el piscardo con Phoxinus bigerri respecto a Phoxinus phoxinus.

En la familia cobítidos, también del orden cipriniformes, se reconocen actualmente tres especies todas ellas endémicas: la lamprehuela (Cobitis calderoni), la colmilleja (C. paludica), anteriormente también citada como C. maroccana, y la recientemente descrita (DOADRIO Y PERDICES, 1997) colmilleja del Alagón (C. vettonica).

En los ciprinodontiformes dos hechos conviene señalar: el reconocimiento de la familia Valenciidae, en la que se encuadra el samaruc (Valencia hispanica), y la descripción de una nueva especie, el salinete (Aphanius baeticus) que recientemente ha sido segregado del fartet (Aphanius iberus) y es exclusivo de los ríos que vierten al golfo de Cádiz.

\section{ESTADO DE CONSERVACIÓN DE LA ICTIOFAUNA AU- TÓCTONA}

\section{III.1. Un predominio cada vez más acusado de las especies amenazadas}

La Lista Roja de los Vertebrados de España de 1986 incluye 32 especies autóctonas, excluyendo las nueve de carácter fundamentalmente marino. En ellas figuran tres especies en peligro (EN), seis vulnerables (VU), dos raras (R), una en peligro indeterminado (I), dos como insuficientemente conocidas (K) y dieciocho no amenazadas (NA). La situación podría parecer relativamente buena o poco preocupante, pues las no amenazadas eran más que las que presentaban algún tipo de amenaza 18 frente a 14, pero esta apreciación respondía más a un insuficiente conocimiento de la realidad que a un estado de conservación relativamente satisfactorio.

Sólo seis años después el Libro Rojo (1992) refleja una situación bastante diferente. Reconoce 42 especies de las cuales hay: una extinguida, seis en peligro, doce vulnerables, nueve raras, una insuficientemente conocida y catorce no amenazadas. Se ha pasado por tanto de que las no amenazadas fueran mayoría, el $56 \%$, a sólo un tercio, el $33 \%$. Como los criterios de valoración del estado de conservación son los mismos en ambas fuentes y el número de especies ha aumentado en diez puede pensarse que esta es la causa principal del empeoramiento pero la realidad es más compleja, como refleja el CUADRO 2, y que merece un breve análisis por categorías. 
Cuadro 2. Comparación de los datos de categorías de conservación de los peces fluviales en 1986 y 1992.

\begin{tabular}{cccccccccc}
\hline & $E X$ & $E N$ & $V U$ & $R$ & $I$ & $K$ & $N A$ & [n.cit] & Total 1986 \\
\hline$E N$ & & 5 & & & & & & & 5 \\
$V U$ & & & 6 & & & & & & 6 \\
$R$ & & 1 & & 1 & & & & & 1 \\
$I$ & & & 1 & & & & & & 2 \\
$K$ & & & & 1 & & 1 & 9 & 1 & 18 \\
$N A$ & & & 5 & 3 & & & 5 & & 9 \\
[n.cit] & 1 & & & 3 & & & 14 & 1 & \\
Total 1992 & 1 & 6 & 12 & 8 & 0 & 1 & \\
\hline
\end{tabular}

Elaborado a partir de la lista roja de los vertebrados españoles (ICONA 1986) y del Libro rojo de los vertebrados españoles (BLANCO y GoNZÁLEZ 1992). Los datos de 1986 en filas y los de 2001 en columnas. Las categorías son: EX: extinta, EN: en peligro, VU: vulnerable, R: raro, I: peligro indeterminado, K: insuficientemente conocido, NA: no amenazado, [n.cit] no citado.

Que en 1986 no haya especies extinguidas y en 1992 haya una no significa un empeoramiento ya que se trata de la lamprea de río no incluida en la Lista Roja de 1986 y extinguida con anterioridad a esta fecha como hemos visto.

El paso de 5 especies en peligro en 1986 a 6 en 1992 sí entraña un ligero empeoramiento ya que hay una especie, el jarabugo, catalogada en 1986 como rara que en 1992 pasa a ser considerada en peligro, lo que si significa un empeoramiento. Las otras cinco especies en peligro comunes a ambas fuentes son el fraile, el cavilat, el esturión, el samaruc y el fartet.

El paso de 6 a 12 especies vulnerables de 1986 a 1992 sí significa un claro empeoramiento en la valoración de su conservación, pues las especies afectadas figuran en ambas fuentes. Todas las especies consideradas vulnerables en 1986 lo son también en 1992. Se trata del sábalo, la saboga, la anguila, el salmón, la lamprehuela y el espinoso. De las otras seis especies una, la lamprea marina, estaba considerada como insuficientemente conocida, probablemente por lo reciente de su descripción, y las otras cinco como no amenazadas. Son la trucha común, los barbos comizo y de montaña, el bagre y la colmilleja.

De las ocho especies consideradas como raras en 1992 la única considerada como tal en 1986 mantiene esta valoración. Tres no aparecen citadas en 1986 por lo que en principio no indican ni mejora ni empeoramiento. Las otras cuatro sí implican, sin embargo, una valoración más negativa. El barbo cabecicorto pasa de insuficientemente conocido a raro y los otros tres, el barbo colirrojo, la pardilla y la loina, de no amenazadas a raras. En conjunto se manifiesta un claro empeoramiento.

No hay especies catalogadas en peligro indeterminado, la única que mereció tal consideración en 1986, la lamprea marina, pasa a vulnerable, y la bo- 
gardilla, única que está considerada como insuficientemente conocida, mantiene su status.

Por lo que se refiere a las no amenazadas de las catorce así calificadas en 1992 nueve también lo eran en 1986 y cinco son especies no recogidas en esta fecha.

Si efectuamos la comparación inversa vemos que de las 32 especies reconocidas en 1986 no hay ningún cambio que implique una mejora y sí varios que indican un empeoramiento. Así la lamprea marina pasa de peligro indeterminado a vulnerable, el barbo cabecicorto de insuficientemente conocido a raro, el jarabugo de raro a en peligro y el barbo cabecicorto, la pardilla y la loina de no amenazado a raro. Por tanto casi un $20 \%$ de las especies, 6 de 32, han empeorado su calificación.

Esto no significa necesariamente que este deterioro se haya producido en su totalidad, aunque probablemente sí en parte, en esos años, sino que el progreso investigador ha permitido conocer mejor una situación que era peor de lo que se pensaba, por insuficiente conocimiento de la situación.

La comparación de los datos de 1992 y los de 2001 puede permitir arrojar algo de luz sobre este problema. El Atlas de 2001 incorpora tres especies ausentes del Libro Rojo de 1992: la colmilleja del Alagón de descripción reciente (EN), la boga del Guadiana anteriormente subespecie de la boga de río (VU) y la aguja de río no incluida en el Libro Rojo por considerarla principalmente marina (LR). Su diferente estado de conservación no influye de modo sensible en el conjunto de nuestra ictiofauna y por ello el notable empeoramiento global que se observa entre ambas fuentes resulta del mayor interés para intentar valorar hasta qué punto se debe a un deterioro real o a los cambios en los criterios de calificación. Parece por tanto conveniente analizar las cuarenta y dos especies comunes a ambas listas. El cuadro adjunto presenta una síntesis de estos cambios.

En 1992 se señalaban 6 especies en peligro y en 2001 la suma de especies en peligro y en peligro crítico asciende a 13. Dos de las tres especies en peligro crítico en 2001, el esturión y el cavilat, estaban ya en peligro y la tercera, la lamprea de arroyo, estaba considerada como rara. En estos tres casos los cambios en su catalogación, más que un empeoramiento, indican simplemente el reconocimiento con una nueva categoría de una situación tan crítica ya con anterioridad que un empeoramiento supondría la extinción. Las otras cuatro especies consideradas en peligro en 1992, jarabugo, fartet, samaruc y fraile, mantienen esa consideración en 2001 y hay cinco que empeoran su situación pues no lo estaban en el Libro Rojo y sí en el Atlas. Se trata de dos 
consideradas antes vulnerables, el salmón y el espinoso, dos raras, la loina y la madrija, y una rara, la bogardilla.

Cuadro 3. Comparación de los datos de categorías de conservación de los peces fluviales en 1992 y 2001.

\begin{tabular}{cccccccccc}
\hline & $E X$ & $E N$ & $V U$ & $R$ & $I$ & $K$ & $N A$ & [n.cit.] & Total 1992 \\
\hline$R E$ & 1 & & & & & & & & 1 \\
$C R$ & & 2 & & 1 & & & & & 3 \\
$E N$ & & 4 & 2 & 2 & & 1 & & 1 & 10 \\
$V U$ & & & 10 & 5 & & & 8 & 1 & 24 \\
$L R$ & & & & & & & 6 & 1 & 7 \\
Total 2001 & 1 & 6 & 12 & 8 & 0 & 1 & 14 & 3 & \\
\hline
\end{tabular}

Elaborado a partir del Libro rojo de los vertebrados españoles (BLANCO y GONZÁLEZ 1992) y del Atlas y Libro rojo de los peces continentales de España (DOADRIO, 2001). Los datos de 1992 en columnas y los de 2001 en filas. Las categorías para 1992 son: EN: en peligro, VU: vulnerable, R: raro, I: peligro indeterminado, K: insuficientemente conocido, NA: no amenazado, n.cit.: no citados. Las categorías de 2001 son las de la UICN: RE: extinto regional, CR: en peligro crítico, EN: en peligro, VU: vulnerable, LR/NT: bajo riesgo no amenazada.

Nada menos que 24 especies están catalogadas como vulnerables en 2001. De ellas 10 tenían esta misma consideración en 1992: la lamprea marina, la saboga, el sábalo, la anguila, la trucha común, los barbos común, comizo y de montaña, el bagre, la lamprehuela y la colmilleja. Otras 5 especies estaban consideradas raras: los barbos cabecicorto, mediterráneo y colirrojo, la pardilla y el bordallo. Nada menos que 8 pasan de no amenazadas a vulnerables: la bermejuela, la boga del Duero, el gobio, el piscardo, el calandino, el lobo de río, el pejerrey y el cacho.

Finalmente, solo media docena de especies no presentan mayores problemas de conservación en 2001. Todas ellas no amenazadas en 1992. Se trata de los barbos común, de Graells y gitano, la madrilla, la boga de río y la tenca. La situación ha empeorado por tanto notablemente pues las especies no amenazadas han bajado de 14 a sólo 6 .

Si tenemos en cuenta que las seis descripciones recientes de nuevas especies endémicas han sido ubicadas por sus descubridores/descriptores en las categorías de mayor amenaza, puede afirmarse que los riesgos y el deterioro global se acentúan. En efecto tres de ellas han sido catalogadas en peligro critico: la pardilla oretana y los cachos malagueño y del Gallo, y las otras tres en peligro: la sarda, el cacho levantino y el salinete.

El mal estado de conservación y progresivo deterioro, al menos estadístico de nuestra ictiofauna fluvial queda claramente de manifiesto con estos datos. A ello ha contribuido, sin duda, un mayor rigor en la definiciones de las categorías de conservación de la UICN usadas en la actualidad, respecto a las tradicionales, pero es también indudable que la situación real ha empeorado 
y a ello han contribuido y siguen contribuyendo infraestructuras hidráulicas, manipulación de caudales, vertidos y contaminación de las aguas, sobreexplotación de acuíferos y también introducción de especies exóticas, translocaciones de especies y genotipos, sobrepesca, etc...

Por todo ello parece conveniente efectuar un breve análisis de los integrantes de las diferentes categorías de conservación.

Cuadro 4. Lista de los peces continentales españoles y de su estado de conservación.

\begin{tabular}{|c|c|c|c|c|c|c|}
\hline Nombre científico & Nombre vulgar & ** & 1986 & 1992 & 2001 & 2011 \\
\hline Lampetra fluvialtilis & lamprea de río & & {$[-]$} & EX & $\mathrm{RE}$ & $\mathrm{RE}$ \\
\hline Lampetra planeri & lamprea arroyo & & $\mathrm{R}$ & $\mathrm{R}$ & $\mathrm{CR}$ & $\mathrm{CR}$ \\
\hline Petromizon marinus & lamprea marina & & I & VU & VU & VU \\
\hline Acipenser sturio & esturión & & $\mathrm{EN}$ & EN & $\mathrm{CR}$ & CR \\
\hline Alosa alosa & sábalo & & V & VU & VU & VU \\
\hline Alosa fallax & saboga & & V & VU & VU & VU \\
\hline Anguilla anguilla & anguila & & V & VU & VU & VU \\
\hline Salmo salar & salmón & & $\mathrm{V}$ & VU & EN & EN \\
\hline Salmo trutta & trucha común & & NA & VU & VU & VU \\
\hline Anaecypris hispanica & jarabugo & ** & $\mathrm{R}$ & EN & EN & EN \\
\hline Luciobarbus bocagei & barbo común & ** & NA & NA & LR & LR \\
\hline Luciobarbus comizo (1) & barbo comiza & $* *$ & NA & VU & VU & VU \\
\hline Luciobarbus microcephalus & barbo cabecicorto & $* *$ & $\mathrm{~K}$ & $\mathrm{R}$ & VU & VU \\
\hline Barbus graellsi & barbo de Graells & & {$[-]$} & NA & LR & LR \\
\hline Barbus guiraonis & barbo mediterraneo & $* *$ & {$[-]$} & $\mathrm{R}$ & VU & VU \\
\hline Barbus haasi. & barbo colirrojo & $* *$ & NA & $\mathrm{R}$ & VU & VU \\
\hline Barbus meridionalis & barbo montaña & & NA & VU & VU & VU \\
\hline Barbus sclateri & barbo gitano & $* *$ & {$[-]$} & NA & LR & LR \\
\hline Achondrostoma salmanticum & sarda & $* *$ & {$[-]$} & {$[-]$} & {$[-]$} & $\mathrm{EN}$ \\
\hline Achondrostoma arcasii & bermejuela & $* *$ & NA & NA & VU & VU \\
\hline Iberochondrostoma oretanum & pardilla oretana & $* *$ & {$[-]$} & {$[-]$} & {$[-]$} & $\mathrm{CR}$ \\
\hline Iberochondrostoma lemmingii & pardilla & $* *$ & NA & $\mathrm{R}$ & VU & VU \\
\hline Parachondrostoma turiense & madrija & $* *$ & {$[-]$} & $\mathrm{R}$ & EN & EN \\
\hline Parachondrostoma arrigonis & loina & $* *$ & NA & $\mathrm{R}$ & EN & EN \\
\hline Parachondrostoma miegii & madrilla & $* *$ & {$[-]$} & NA & LR & LR \\
\hline Pseudochondrostoma willkommii & boga del Guadiana & $* *$ & {$[-]$} & {$[-]$} & VU & VU \\
\hline Pseudochondrostoma duriense. & boga del Duero & $* *$ & {$[-]$} & NA & VU & VU \\
\hline Pseudochondrostoma polylepis & boga del Tajo & $* *$ & NA & NA & LR & LR \\
\hline Gobio gobio $=$ lozanoi & gobio & & NA & NA & VU & VU \\
\hline Phoxinus phoxinus & piscardo & & NA & NA & VU & VU \\
\hline Squalius alburnoides & calandino & $* *$ & NA & NA & VU & VU \\
\hline Squalius caroliterti & bordallo & $* *$ & [na] & $\mathrm{R}$ & $\mathrm{vu}$ & VU \\
\hline Squalius cephalus & bagre & & NA & VU & $\mathrm{vu}$ & VU \\
\hline Squalius palaciosi & bogardilla & $* *$ & $\mathrm{~K}$ & $\mathrm{~K}$ & EN & $\mathrm{EN}$ \\
\hline Squalius malacitanus & cacho malagueño & $* *$ & [na] & {$[-]$} & {$[-]$} & $\mathrm{CR}$ \\
\hline Squalius castellanus & caho del Gallo & $* *$ & {$[-]$} & {$[-]$} & {$[-]$} & CR \\
\hline Squalius valentinus & cacho levantino & $* *$ & [na] & {$[-]$} & {$[-]$} & $\mathrm{EN}$ \\
\hline Squalius pyrenaicus & cacho & $* *$ & [na] & NA & VU & VU \\
\hline
\end{tabular}




\begin{tabular}{|c|c|c|c|c|c|c|}
\hline Tinca tinca & tenca & & NA & NA & LR & NT \\
\hline Cobitis calderoni & lamprehuela & ** & $\mathrm{V}$ & VU & VU & VU \\
\hline Cobitis paludica (2) & colmilleja & ** & NA & VU & VU & VU \\
\hline Cobitis vettonica & colmilleja Alagón & $* *$ & [na] & {$[-]$} & $\mathrm{EN}$ & $\mathrm{EN}$ \\
\hline Barbatula barbatula & lobo de río & & $\mathrm{NA}$ & NA & VU & VU \\
\hline Aphanius Iberus & fartet & & $\mathrm{EN}$ & $\mathrm{EN}$ & $\mathrm{EN}$ & $\mathrm{EN}$ \\
\hline Aphanius baeticus & salinete & $* *$ & {$[-]$} & {$[-]$} & {$[-]$} & $\mathrm{EN}$ \\
\hline Valencia hispanica & samaruc & $* *$ & EN & EN & EN & $\mathrm{EN}$ \\
\hline Atherina boyeri & pejerrey & & NA & NA & VU & VU \\
\hline Gasterosteus gymnurus & espinoso & & $\mathrm{V}$ & VU & EN & EN \\
\hline Cottus gobio & cavilat & & {$[-]$} & EN & $\mathrm{CR}$ & $\mathrm{CR}$ \\
\hline Salaria fluviatilis & fraile & & {$[-]$} & EN & EN & EN \\
\hline Syngnathus abaster & aguja de río & & NA & {$[-]$} & LR & LR \\
\hline
\end{tabular}

Las fechas de las cuatro últimas columnas indican la fecha de la fuente: Lista Roja para 1986, Libro Rojo para 1992, Atlas y Libro Rojo para 2001. Para 2011 son las mismas de 1992 añadiendo las propuestas por los autores para las descritas posteriormente. La columna del doble asterisco $(* *)$ indica las especies endémicas de la península ibérica.

(1) incluye barbus steindacheri, (2) citado como cobitis marocana en 1992 e incluye $c$. haasi 1992.

\section{III.2. Especies en peligro crítico}

Tres especies figuran en el Atlas como en peligro critico: la lamprea de arroyo (Lampetra planeri), el esturión (Accipenser sturio) y el cavilat (Cottus gobio). A ellas habría que añadir otras tres descritas recientemente: la pardilla oretana (Chondrostoma oretanum), el bordallo o cacho del Gallo (Squalius castellanus) y el cachuelo malagueño (Squalius malacitanus). Cada una de ellas merece un comentario por separado.

La presencia en España de la lamprea de arroyo se limita al río Olavidea en la vertiente francesa del Pirineo Navarro. Por esta reducida área de presencia fue clasificada como rara (R) en 1986 y 1992 y como en peligro critico (CR) de acuerdo con los criterios B1+2ce. Su situación a nivel mundial es más favorable y si en 1986 en la Lista Roja se señala que en Europa está considerada como vulnerable, en 2001 se recoge que la UICN la cataloga como LR/NT (bajo riesgo/no amenazada). Su aparente empeoramiento en España, al pasar de rara a en peligro critico, se debe más a la aplicación de nuevos criterios que dan más importancia que en el pasado a la extensión del área de presencia que a un deterioro real de su situación. Esta se limita a una sola cuadrícula, por lo que es necesario un seguimiento de las actuaciones de todo tipo que pudieran poner en peligro la supervivencia de la única población actual de esta especie en nuestro país.

Del esturión existen citas en bastantes ríos peninsulares y fue relativamente abundante en el bajo Guadalquivir donde abasteció hasta 1967 una modesta fábrica de caviar (DOADRIO et AL., 1991). Hoy se encuentra en peligro crítico tanto a nivel español como a nivel mundial (criterio A2d). El Atlas de 
2001 señala su presencia en el bajo Guadalquivir, pero indicando la ausencia de capturas en los últimos años.

El cavilat merece una atención especial. Considerado en peligro en 1986 y 1992 pasa a ser clasificado como en peligro crítico en 2001. Sin embargo en las bases de datos del Ministerio figura como simplemente en peligro. Su presencia actual es algo mayor que las dos anteriores pues sobrevive en el valle de Arán y en las cabeceras de varios ríos del norte de Navarra. Sin embargo su presencia territorial sigue siendo reducida y la falta de conexión con otras poblaciones europeas, donde no está catalogada como amenazada, parecen apoyar el criterio del Atlas de 2001 de considerarla en peligro critico (CR B1+2bcde). Recientemente se han propuesto cambios taxonómicos y nomenclaturales para esta (FREYHOF et $A L$., 2005). De acuerdo con ellos, las poblaciones del Valle de Arán y de Navarra podrían pertenecer a especies diferentes: $C$. hispanionensis y $C$. aturi respectivamente. Desde una óptica conservacionista estos cambios no harían sino reforzar su consideración en peligro crítico, pues si ya se les asignaba conjuntamente, con mayor razón la merecerían por separado.

La pardilla oretana (Iberochondrostoma oretanum) es una especie recientemente descrita que debe considerarse endémica del río Robledillo, afluente del río Jándula en la cuenca del río Guadalquivir (DOADRIO y CARMONA, 2003), pues la población del río Fresneda parece estar introgredida genéticamente con Chondrostoma lemmingii (DOADRIO y CARMONA, 2009). Tan reducida área y la fuerte disminución de su población en los últimos años y las obras que se realizan para la construcción de un dique en el río Robledillo, que acarrearía la destrucción del bosque de ribera en cuyas raíces encontraba refugio, y a la modificación del caudal suponen una grave amenaza y obligan calificar su estado de conservación como en peligro critico $\mathrm{CR}$ de acuerdo con los criterios B1+2ce.

El bordallo del gallo o cacho del Gallo (Squalius castellanus) ha sido descrito muy recientemente (DOADRIO et $A L$., 2007) en la cuenca del río Gallo, afluente del Tajo en su cuenca alta y de sus tributarios Bullombes y Arandilla. Lo reducido de su área y de sus poblaciones y la contaminación de las aguas por los vertidos de Molina de Aragón aconsejan según sus descriptores asignarle la calificación de en peligro critico (CR B1 + 2ce)

El cacho malagueño Squalius malacitanus, descrito hace sólo un lustro (DOADRIO y CARMONA 2006), es conocido solamente de los ríos Guadalmina y Genal, afluente de Guadiaro, y aunque es probable que pueda estar presente en algún otro río malagueño su reducida área de distribución y las características de estos ríos de régimen muy irregular y situados en una de las 
fachadas costeras más pobladas de la península, cuya demanda creciente de agua hace bajar el nivel de los acuíferos, hacen aconsejable considerarlo según sus descubridores y descriptores en peligro critico (CR). Es un pez muy pequeño, que no alcanza los $8 \mathrm{~cm}$, propio de ríos con tramos de corriente moderada, fondos de grava y aguas claras. En el Guadalmina es el único pez autóctono y en el Genal comparte el habitat con otras especies autóctonas como Barbus sclateri y Chondrostoma wilkommii y también con Anguilla anguilla y Atherina boyeri.

\section{III.3. Especies en peligro}

Solo dos de las especies hoy consideradas en peligro figuraban con esta calificación en 1986 y ambas de distribución levantina: el fartet (Aphanius iberus) y el samaruc (Valencia hispanica). En 2001 su número se elevaba a diez y en la actualidad con las nuevas descripciones a trece. Son las siguientes:

El salmón (Salmo salar) había venido siendo considerado vulnerable tanto en 1986 como en 1992, pero los autores del Atlas de 2001 consideran que debe pasar a estar catalogado en peligro por la fuerte disminución de sus capturas, que en el periodo 1991-98 bajaron de 2.600 ejemplares menos de 1.000 y en la actualidad no llegan a 500. Esta disminución justifica el cambio de catalogación de vulnerable a en peligro (criterio A1bd). La conservación de esta migratoria anádroma exige tomar y mantener medidas eficaces para favorecer su reproducción en los ríos y limitar sus capturas en el mar. Un riesgo adicional son los posibles efectos negativos del cambio climático, menor nivosidad y aumento de la temperatura del agua, sobre la reproducción de la especie y el desarrollo de los alevines (GARCÍA CODRÓN et $A L$., 2010).

El jarabugo (Anaecypris hispanica $\mathrm{B} 1+2 \mathrm{ce}$ ) es un ciprínido de pequeño tamaño que no suele llegar a $10 \mathrm{~cm}$, propio de ríos de corriente lenta, con vegetación sumergida y fondo pedregoso, considerado endémico de la cuenca del Guadiana, aunque fue citado también en el Bembézar (BARRACHINA et AL. 1989) de donde parece haber desaparecido (DOADRIO, 2001). Calificado como raro (R) en la Lista Roja, figura ya como en peligro (EN) en el Libro Rojo de 1992, status que tiene también en Portugal, y a nivel de catálogos autonómicos en Extremadura, pero no en Castilla-La Mancha donde está considerado vulnerable (VU). Lo reducido y disperso de sus poblaciones lo hace especialmente sensible a la contaminación y a la depredación por especies introducidas, como la perca americana (Micropterus salmoides) y el pez sol (Lepomis gibbosus), que son sus principales riesgos.

La loina (Parahondrostoma arrigionis) y la madrija (P. turiense) son dos 
ciprínidos de tamaño medio de hasta $30 \mathrm{~cm}$, recientemente independizados (ELVIRA, 1997) de Chondrostoma toxostoma. Su tradicional inclusión en esta especie colectiva que por su amplia distribución no era considerada amenazada en la Lista Roja de 1986, ha retrasado la adecuada valoración de su estado de conservación. Ambas fueron calificadas como raras $(\mathrm{R})$ en el Libro Rojo de 1992 y en el reciente Atlas figuran finalmente como en peligro (EN). Las dos son especies de notable amplitud ecológica, pues aunque precisan aguas vivas para su reproducción, toleran fuera de esa época aguas tranquilas de inferior calidad e incluso embalses. La intensa explotación antrópica de los ríos en que viven estos endemismos, el Júcar para la loina y el Turia y Mijares para la madrija, con embalses, derivaciones para riego, canalizaciones, etc., y la introducción de especies alóctonas piscívoras son los factores responsables de su situación. En el caso de la loina su declive coincide con la introducción de Pseudochondrostoma polylepis en la cuenca del Júcar, ya que este pez autóctono de la cuenca del Tajo es una causa de su declive.

La bogardilla (Squalius palaciosi (=Leuciscus palaciosi, =Iberocypris palaciosi)) presenta, según su descriptor, un singular interés y especial valor biológico por su posible origen híbrido entre Squalius pyrenaicus y $S$. alburnoides. Estos posibles parentales se habían clasificado anteriormente en géneros diferentes, Leuciscus y Tropidophoxinellus, por lo que de haberse mantenido la validez de los mismos habría sido un híbrido intergenérico. De descripción bastante reciente (DOADRIO, 1980), su área de distribución es sumamente restringida con sólo tres poblaciones en la cuenca del Guadalquivir, dos de ellas afectadas por los vertidos industriales de Puertollano y la presa de Encinarejo en el río Jándula. Calificada inicialmente como insuficientemente conocida (Lista Roja de 1986 y Libro Rojo de 1992) y de interés especial (Real Decreto 439/1990) merece actualmente el status de en peligro por lo reducido de su área y la pérdida de efectivos de sus poblaciones (criterios $b 1+2 c)$.

La colmilleja del Alagón (Cobittis vettonica) es otra de las especies recientemente descritas de nuestra ictiofauna, ya que había pasado inadvertida hasta su publicación por Doadrio y Perdices en 1997. El área de este pequeño cobítido se limita a la cuenca del Alagón y sus tributarios. Este reducido areal, sus numerosos predadores, sobre todo en los tramos bajos, y su uso como cebo vivo, la sitúan en una posición delicada que justifica tanto su clasificación actual como especie en peligro de extinción, como el que al año siguiente a su descripción fuera ya incluida en el catálogo de especies protegidas de Extremadura en la categoría de "sensible a la alteración del hábitat". 
El fartet (Aphanius iberus) es un ciprinodóndido euriahalino de muy pequeño tamaño, propio de charcas, lagunas salinas, desembocaduras y cursos de agua dulce disperso por las zonas costeras mediterráneas y algunas lagunas interiores, cuyas poblaciones del golfo de Cádiz han sido consideradas recientemente como especie nueva con el nombre de Aphanius baeticus (DOADRIO et AL. 2002). Si la desaparición de buena parte de sus poblaciones por deterioro del hábitat por actuaciones hidráulicas y la predación de especies introducidas como el fúndulo y la gambusia obligaba a calificarlo de en peligro, el reparto de su ya reducida área conjunta en dos especies aconsejaría su paso a la categoría de en peligro critico sobre todo para Aphanius baeticus cuya área se reduce casi exclusivamente a Doñana y el entorno de la bahía gaditana.

El samaruc (Valencia hispanica) es un ciprinodontiforme endémico también calificado en peligro de extinción. Se trata también de un ciprinodóntido de muy pequeño tamaño, pues no suele llegar a $8 \mathrm{~cm}$, propio de lagunas, albuferas y acequias del golfo de Valencia desde Denia al Delta del Ebro. En la actualidad sobreviven ocho poblaciones de las que sólo cuatro parecen tener efectivos suficientes o abundantes (DOADRIO, 2001). Este hecho, la continuada reducción y deterioro de su hábitat y sus numerosos enemigos y competidores, entre los que se encuentra un pez aún más pequeño, como es la gambusia (Gambusia holbrooki), justifican su crítica situación.

El espinoso es el único representante español de la familia de Gasterosteidae que se había tradicionalmente incluido en Gasterosteus aculeatus, pero que según KotTELAT (1997) debe incluirse en Gasterosteus gymnurus. Disperso por Galicia litoral e interior y áreas costeras mediterráneas de Denia a Gerona y con dos localidades en el País Vasco, su reducida área total y lo disperso de sus poblaciones justifican su consideración como especie en peligro. Esto supone una situación de mayor amenaza respecto a obras anteriores, pues en 1986 y 1992 figuraba como vulnerable. A ello han contribuido, entre otros factores, la construcción de embalses, la desecación de lagunas y marjales y la contaminación por vertidos industriales y urbanos.

El fraile (Salaria fluviatilis) es un blénido bentónico propio de cauces lentos desde el alto Ebro y Gerona hasta Andalucía. Pese a su área relativamente amplia la fuerte reducción de muchas de sus poblaciones y la desaparición de otras tanto por obras hidráulicas varias como por predación de especies exóticas entre la se destacan la perca americana (Micropterus salmoides), el lucio (Esox lucius) y el pez gato (Ictalurus melas) justifica su catalogación como en peligro, status que ya tenía en el libro rojo. 
El cachuelo valenciano o bagra (Squalius valentinus) ha sido descrito aun más recientemente (DOADRIO y CARMONA, 2006) y constituye un nuevo endemismo de los ríos levantinos entre Castellón y el sur de Alicante: Mijares, Turia, Júcar, Serpis, Bullent, Gorgos, Guadalest, Monebre y Vinalopo y también en la Albufera de Valencia. En ríos como el Júcar, que cuentan con poblaciones de cacho y bagra, sus aéreas no son sin embargo alopátricas pues el cacho prefiere los tramos bajos y el cachuelo valenciano los altos de corriente moderada, aguas claras y fondo gravoso. En otros ríos importantes de la región es simpátrico con otras especies autóctonas como Barbus guiraonis, Parachondrostoma turiense, pero en los ríos de menor longitud como el Gorgos es la única especie presente. Actividades humanas con impacto hidrológico y especies introducidas son responsables de su escasez que obliga a considerarla en peligro (EN B12c) según los criterios de la IUCN.

La sarda (Achondrostoma salmanticum) es una especie de reciente descripción (DOADRIO y ELVIRA, 2007), con un reducido rango de distribución. Este se limita a los ríos Águeda, Huebra, Turones y Uces, afluentes del Duero. Hasta principios de la década de los 90 también se pescaban sardas en el embalse de Ricobayo, en el río Esla en Zamora, pero desde entonces han desaparecido. Este nuevo ciprínido es considerado En Peligro (EN) de acuerdo a las categorías de la Lista Roja de la UICN.

\section{III.4. Especies vulnerables.}

Las especies calificadas de vulnerables se elevan a trece, lo que aconseja un tratamiento menos individualizado, más sintético, que se centrará en tres grupos o situaciones.

La lamprea marina (Petromizon marinus) fue inicialmente calificada como en peligro indeterminado (Lista Roja de 1986), pero ya en el Libro Rojo (1992) y también en el Atlas (2001) se cambia esta calificación a la de vulnerable (VU B1 + 2abcd). Este cambio parece deberse principalmente a la disminución de su presencia, que actualmente se reduce casi exclusivamente a las costas cantábricas, gallegas y del golfo de Cádiz, ya que en el Mediterráneo está casi extinguida, pues sus citas recientes se reducen a las proximidades del Estrecho y al delta del Ebro. Por otra parte su presencia en el interior se mantiene sólo en el Guadiana, aguas debajo de Mérida, aunque es muy escasa por lo que en Extremadura está considerada en peligro de extinción.

Los dos clupeidos presentes en España, el sábalo (Alosa alosa) y la saboga (Alosa fallax) están también calificados como vulnerables. Ello está plenamente justificado por su presencia, cada vez más reducida, que en el primero 
se limita al curso inferior de unos pocos ríos en las costas cantábricas y atlánticas y al Guadiana pacense y en el segundo al sur de Galicia curso bajo del Guadiana y Guadalquivir, delta del Ebro y golfo de Rosas. Se trata de especies migradoras anádromas, principalmente marinas, sometidas a una fuerte sobrepesca y muy perjudicadas en sus posibilidades de ascenso río arriba por la construcción de presas.

La anguila (Anguilla anguilla) es una especie migradora catadroma cuyas larvas o alevines se desplazan desde el Mar de los Sargazos hasta las costas europeas y penetran en los ríos donde se transforman en adultos pudiendo llegar a alcanzar 1,5 m de longitud. Especie omnívora. eurihalina y bentónica vive tanto en aguas marinas, como en salobres y dulces de deltas, albuferas, estuarios, ríos y humedales interiores. A principios del siglo pasado era especie muy abundante en la mayor parte de las cuencas, pero sus poblaciones han venido experimentando una acusada disminución por sobrepesca, degradación y destrucción de humedales, contaminación de ríos y estuarios y para su penetración río arriba por la construcción de embalses presas y derivaciones. Todo ello justifica su consideración como especie vulnerable (VU $2 \mathrm{~cd})$.

La trucha común (Salmo trutta) es una especie propia de aguas frías, rápidas claras y bien oxigenadas, ampliamente distribuida por la Península que pese a ello está considerada como vulnerable (VU 1cde), aunque en la Lista Roja de 1986 se la calificaba como no amenazada. A esta peor consideración de su conservación contribuye no solo la sobrepesca y la contaminación de numerosos ríos y en ocasiones la predación por especies como el lucio (Esox lucio), sino también las consecuencias de un manejo no siempre adecuado por razones de rentabilidad piscícola. Dado el interés deportivo y económico de su pesca son frecuentes las repoblaciones con alevines de otras procedencias con el consiguiente riesgo de contaminación genética. En general se estima que la introgresión genética entre un 5 y un $10 \%$ y que esta parece ser mayor en el centro peninsular.

Cinco son los barbos que merecen ser considerados vulnerables. Dos de ellos se encuadran en el nuevo género Luciobarbus, los barbos comizo y cabecicorto, y los otros tres en el género Barbus, los barbos mediterráneo, cabecicorto y de montaña. Ninguno de ellos era considerado amenazado en la Lista Roja (1986). En el libro Rojo (1992) hay dos catalogados como vulnerables, los barbos comizo y cabecicorto y los otros tres como raros. No cabe, pues, duda alguna de la valoración conservacionista de los barbos ha empeorado.

El barbo comizo (Luciobarbus comizo) es un barbo que puede llegar a al- 
canzar el metro de longitud lo que le convierte en el mayor de los barbos. Endémico de las cuencas del Tajo y Guadiana sus poblaciones se encuentran en regresión y se prevé que en los próximos años la disminución de sus efectivos supere el $20 \%$ lo que justifica que su consideración haya pasado de no amenazado en 1986 y 1992 a vulnerable (VU A2ce) en 2001. Las causas residen en la contaminación de los ríos por los vertidos agrícolas e industriales y la construcción de infraestructuras hidráulicas, presas, derivaciones, etc.

El barbo cabecicorto (Luciobarbus microcephalus) es un barbo de mediano tamaño, hasta $50 \mathrm{~cm}$ endémico de la cuenca del Guadiana. Al compartir área y hábitat con el barbo comizo, aunque es capaz subir con mayor frecuencia que él a los cursos altos, las causas de su regresión son semejantes y de su catalogación como especie vulnerable las mismas (VU A2ce). En 1986 estaba considerado insuficientemente conocido y en 1992 como raro.

El barbo mediterráneo (Barbus guiraonis) es un endemismo levantino de tamaño medio que penetra en la cuenca del Guadiana en el alto Cigüela. Propio de cursos medios y bajo de los ríos y también presente en embalses y lagunas, su mal estado de conservación (VU A2ce) se debe principalmente al impacto de especies exóticas como el pez sol (Lepomis gibbosus), el pez gato (Amelurus melas), la perca americana (Micropterus salmoides), el lucio (Esox lucio) y el luciperca (Sander lucioperca).

El barbo colirrojo (Barbus haasi) es un barbo endémico de pequeño tamaño propio de la cuenca del Ebro aunque su área se extiende también a gran parte de la Cataluña litoral hasta el alto Ter y por el sur hasta el Palancia en Castellón y alto Turia en Teruel-Valencia. Propio de las cuencas altas de los ríos las causas de su deficiente conservación son semejantes a las del anterior y su catalogación (VU A2ce).

También el barbo de montaña (Barbus meridionalis) es un barbo de pequeño tamaño que prefiere cursos altos de aguas frías y limpias aunque también puede descender a tramos medios y bajos de aguas más cálidas y lentas. Su presencia española se limita a Cataluña al noreste del Llobregat. A su declive parecen contribuir especialmente las obras hidráulicas, derivaciones y la contaminación, lo que justifica su consideración como vulnerable (VU A2ce).

Dentro del grupo Chondrostoma, que comprende los antiguos géneros Chondrostoma y Rutilus, son cuatro las especies catalogadas como vulnerables: las bogas del Duero y del Guadiana anteriormente incluidas en Chondrostoma polylepis y hoy encuadradas en el nuevo género Pseudochondros- 
toma, y la bermejuela y la pardilla consideradas no amenazadas en 1986 y 1992.

La boga del Duero (Pseudochondrostoma duriense) es un endemismo ibérico de la cuenca del Duero y del Noroeste peninsular descrito recientemente (COELHO, 1985) y antes incluido en Chondrostoma polylepis como ya se ha señalado. Las causas de la tendencia regresiva de las poblaciones de esta especie de mediano tamaño son las habituales.

La boga del Guadiana (Pseudochondrostoma willkommii) un endemismo ibérico de tamaño medio cuya área se extiende por la cuencas del Guadiana, Guadalquivir y parte occidental del mediterráneo andaluz. Pez gato (Amelurus melas), black-bass o perca americana (Micropterus salmoides), lucio (Esox lucius) y lucioperca (Sander lucioperca) parecen ser las causas principales causas del decline de esta especie y también de la boga del Duero.

La bermejuela (Achondrostoma arcasi, =Rutilus arcasii) es un pececillo que puede llegar hasta los $10 \mathrm{~cm}$ de longitud propia de ríos, lagos y lagunas de montaña y altas parameras. Contaminación, obras hidráulicas y principalmente predación por especies introducidas son las causas principales de que su población, aunque aun localmente abundante sobre todo en la vertiente mediterránea, estén en franca regresión y obliguen a considerarla vulnerable (VU A2ce).

La pardilla (Iberochondrostoma lemmingii) en su acepción tradicional se extendía desde el oeste de Salamanca al golfo de Cádiz y la calificación global era vulnerable, pero señalando que las poblaciones de Sierra Morena cordobesa y jienense y de la cuenca del Duero salmantina se consideran en peligro. Conviene destacar que las poblaciones salmantinas de pardilla, según Doadrio, merecían ser tratadas como especie diferente (DOADRIO, 2001). Esto se ha materializado recientemente (DOADRIO Y ELVIRA, 2007) en la descripción de las nuevas especies denominadas Iberochondrostoma salmantinum e I. oretanum. El complejo pardilla son pues hoy tres especies, todas ellas del nuevo género Iberochondrostoma, con status de conservación diferente: la pardilla s. str., cuya área se extiende desde el Tajo a la parte occidental de la cuenca del Guadalquivir sigue catalogada como vulnerable, la sarda o pardilla salmantina en la parte occidental de esta provincia, está calificada como en peligro, y la pardilla oretana en la cuenca del Jándula, se considera en peligro crítico.

Con el cacho (Squalius pyrenaicus) sucede algo semejante a con la pardi1la. Su área de distribución, que pese a su nombre no alcanza el Pirineo, cu- 
bre la Península desde el bajo Ebro y la cuenca del Tajo hacia el sur, según el Atlas. Aunque el conjunto de la especie es considerado como vulnerable se señala que cabe distinguir hasta cinco poblaciones diferenciadas, que podrían merecer tratamiento específico y de las cuales dos deberían considerarse en peligro. Una se extiende en el extremo sur de la Península y por los ríos de la vertiente mediterránea andaluza y otra por los ríos del litoral de la región valenciana. Precisamente para estas aéreas se han reconocido dos nuevas especies el cacho o cachuelo malagueño (Squalius malacitanus), considerada en peligro crítico, y cacho levantino o valenciano (Squalius valentinus) calificado como en peligro.

El gobio español (Gobio lozanoi), anteriormente incluido en la amplia especie europea Gobio gobio de la que ha sido recientemente independizado a nivel especie (DOADRIO y MADEIRA, 2004), debe mantener la consideración de vulnerable que tenían anteriormente sus poblaciones. Se trata de una especie que podría calificarse de casi endémica, pues su presencia en el suroeste de Francia parece limitarse a la cuenca del Adour. De pequeño tamaño es sensible a las especies piscívoras introducidas.

Al piscardo español le pasa algo parecido al gobio pues tradicionalmente incluido en Phoxinus phoxinus ha sido recientemente descrito como especie nueva con el nombre de Phoxinus bigerri (KoTTELAT, 2007). La consideración de vulnerable debe considerarse la adecuada a pesar del cambio taxonómico. Se trataría de un endemismo franco-ibérico con una reducida presencia al norte del Pirineo.

El calandino (Squalius alburnoides) a pesar de su amplia área de distribución, que se extiende desde el suroeste de la cuenca del Duero al Guadalquivir y como otras especies de pequeño tamaño, sensible a la predación por especies introducidas y deterioro de su hábitat, está también catalogado como vulnerable.

La segregación de las poblaciones del alto Tajo como bordallo del Gallo (Squalius castellanus) deja al bordallo (Squalius caroliterti) como un endemismo de la cuenca del Duero y Galicia. Esta reestructuración no afecta a su status de conservación, que aunque en 1986 era de no amenazado había pasado a vulnerable en el Libro Rojo de 1992, consideración que sigue manteniendo.

Algo parecido sucede con las poblaciones españolas del bagre. Asignadas tradicionalmente a Squalius cephalus, la descripción de Squalius laietanus (DOADRIO et $A L ., 2007$ ) supone su asignación a esta nueva especie. Como en los casos anteriores esto no afecta a la consideración conservacionista de 
nuestras poblaciones de bagre que deben calificarse como vulnerables desde 1992.

Dos cobítidos aparecen considerados como vulnerables, la lamprehuela (Cobittis calderoni) y la colmilleja (Cobitis paludica). La primera tenía ya esta consideración en la Lista roja de 1986, pero la segunda catalogada en esa fecha como no amenazada bajo la denominación de Cobittis marocana no se considera vulnerable hasta 1992. De ella se han segregado las poblaciones extremeña de la cuenca del Alagón como una nueva especie llamada Cobitis vettonica. En el Libro Rojo (1992) se consideran como especie las poblaciones de valencianas de raboseta como Cobitis haasi calificadas de "peligro indeterminado". Su inclusión posterior en Cobitis paludica implica que también estas poblaciones estén consideradas como vulnerables.

Lobo de río (Barbatula barbatula (=Noemachelius barbatula)) es el único representante en nuestro país de la familia balitóridos. Pese a su nombre, que induciría a pensar en un depredador, es un pez de pequeño tamaño, hasta 20 $\mathrm{cm}$, sensible a la calidad de las aguas y a la presencia de especies introducidas como el pez gato, el siluro, etc., características que lo sitúan en la posición de vulnerable.

Pejerrey (Atherrina boyeri) es un pececillo de pequeño tamaño, hasta 10 $\mathrm{cm}$, preferentemente marino, presente en bastantes desembocaduras y estuarios del sur y este de la Península y con algunas poblaciones dulceacuícolas interiores en el valle del Guadalquivir. La sobrepesca y en aguas interiores la predación por especies exóticas son la causa de que su situación sea la de vulnerable.

\section{III.5. Especies no o poco amenazadas.}

Cada vez son menos las especies autóctonas que pueden considerarse no amenazadas. En 1986 se contabilizaban 13 sobre un total de 32 lo que representa casi el $60 \%$. En 1992 el libro rojo registra como no amenazadas 12 especies, pero sobre un total de 40 lo que representaba el $30 \%$. En la actualidad son sólo 7 las especies que consideradas poco o no amenazadas que representarían solo el $14 \%$ ya que con las nuevas descripciones el número de peces autóctonos se ha elevado a 50.

Se trata de la tenca (Tinca tinca) de presencia dispersa por gran parte de la península, del barbo común (Luciobarbus bocagei) endemismo ibérico de las cuencas del Duero y del Tajo, del barbo de Graells (Barbus graellsii) de las del Ebro, Pirineo Oriental y País Vasco-Cantabria, del barbo gitano (Barbus sclateri), la madrilla (Parachondrostoma miegii) de la del Ebro y cantábrico oriental, de la boga del Tajo (Pseudochondrostoma polylepis) de la cuenca 
que le da nombre y de la aguja de rio (Syngnatus abaster) especie eurihalina propia de arroyos y canales litorales entre los ríos Ebro y Segura.

\section{A MODO DE CONCLUSIÓN: DIFERENTE RIQUEZA Y ESTADO DE CONSERVACION DE LAS DISTINTAS CUEN- CAS HIDROGRÁFICAS.}

La comparación del Atlas y Libro Rojo de 2001 y publicaciones posteriores con la Lista Roja de 1986 y el Libro Rojo de 1992 muestra un claro aumento del número de especies de peces fluviales conocidas en nuestro país, al que ha contribuido la descripción de nuevas especies. Al mismo tiempo destaca también un deterioro del estado de conservación con aumento claro en cifras absolutas y porcentuales de las categorías de mayor grado de amenaza

Desde una óptica geográfica esta sucinta visión de la riqueza y estado de conservación de nuestra ictiofauna autóctona quedaría incompleta si no echáramos una rápida ojeada a algunos rasgos básicos de su distribución territorial. Puede ser interesante e ilustrativo conocer cuáles son las cuencas fluviales más ricas en endemismos y al mismo tiempo cual es la amplitud territorial de los mismos. El cuadro adjunto da una visión sintética de ambos aspectos.

Cuadro 5. Número de especies presentes en distintas cuencas hidrográficas por categorías de conservación

\begin{tabular}{|c|c|c|c|c|c|c|}
\hline & $R E$ & $C R$ & $E N$ & $V U$ & $L R$ & TOTAL \\
\hline Cantábrico & & 2 & 2 & 8 & 2 & 14 \\
\hline Galicia & & 0 & 2 & 9 & 1 & 12 \\
\hline Duero & & 0 & 1 & 10 & 2 & 13 \\
\hline Tajo & 0 & 1 & 1 & 9 & 4 & 15 \\
\hline Guadiana & & & 1 & 14 & 3 & 18 \\
\hline Guadalquivir & & 1 & 3 & 12 & 2 & 18 \\
\hline Sur & & 1 & 2 & 7 & 1 & 11 \\
\hline Segura & & 0 & 1 & 7 & 3 & 11 \\
\hline Júcar & & 0 & 7 & 11 & 3 & 21 \\
\hline Ebro & & 0 & 4 & 14 & 4 & 22 \\
\hline Pirineo Oriental & & 0 & 3 & 7 & 4 & 14 \\
\hline
\end{tabular}

Aunque cada una de las cuencas merecería un análisis detallado, cabría señalar en este aspecto algunas de ellas como casos especialmente interesantes.

Destaca por su riqueza y desfavorable situación en cuanto a su estado de conservación la confederación del Júcar, ya que es la segunda por número de especies pero también la cuenca con más especies en peligro de extinción: la loina, la madrija, el cacho levantino, el samaruc, el espinoso, el fraile y el fartet, de ellas las cuatro primeras endémicas. Además cuenta con cinco endemismos ibéricos vulnerables, los barbos mediterráneo y colirrojo, la ber- 
mejuela, la colmilleja y el cacho y otros seis peces vulnerables no endémicos. Por otra parte, la boga de río ha sido introducida en el alto Júcar con efectos negativos para la ictiofauna autóctona y concretamente para su congénere la loina.

En el extremo contrario es destacable también la pobreza de las confederaciones del Sur y del Segura, en parte explicable por las características hidrológicas de su red fluvial y que afectan al conjunto de su ictiofauna con pocos endemismos y la mayoría de ellos de distribución más bien amplia. Sin embargo la reciente descripción del cacho malagueño ha dotado a la Confederación Sur de una especie en peligro crítico, que unida a las dos en peligro que ya tenía, el fartet y el fraile, le confiere un notable interés conservacionista.

Por lo que se refiere a las cuencas de los grandes ríos meseteños cabe señalar que tiene lugar un aumento de la riqueza en especies y de interés conservacionista de norte a sur aunque las recientes descripciones tienden a compensarlo sin llegar a equipararlas, con nuevos endemismos de área reducida en situación de riesgo. Así la cuenca del Duero es la más pobre en especies, con sólo 13, y cuenta con una sola especie en peligro de descripción reciente y distribución restringida, la sarda. Dos descripciones recientes han dotado al Tajo de una especie en peligro crítico, el cacho del Gallo, y otra en peligro, la colmilleja del Alagón, pero contra lo que pudiera pensarse a primera vista, la mayor riqueza en especies, en endemismos y también el mayor número de especies amenazadas corresponde al Guadiana. Este río cuenta con 18 especies, de ellas 11 endémicas, cuenta con una especie en peligro, el jarabugo, que es un endemismo de su cuenca, y tiene además 14 especies vulnerables.

El Guadalquivir por su parte presenta el interés de ser, tal vez haber sido, el último reducto del esturión y tener tres endemismos gravemente amenazados de área restringida: uno en el entorno del golfo de Cádiz, el salinete, y otros dos en Sierra Morena, la pardilla oretana y la bogardilla. Por otra parte con sus 18 especies, la mitad de ellas endémicas, comparte con el Guadiana el tercer puesto por diversidad.

El Ebro es el río con mayor número de especies autóctonas (22) a lo que contribuye no sólo la extensión de su cuenca sino su diversidad interna que a los distintos ambientes de la mitad norte peninsular une en el delta los propios de las desembocaduras de los grandes ríos y de los medios litorales y sublitorales mediterráneos. Cuenta con cuatro peces en peligro, fartet, samaruc, espinoso y fraile, y catorce vulnerables, pero ninguno en peligro crítico.

Las regiones norteñas, Cantábrico y Galicia, destacan por su pobreza relativa en especies y escasez de endémicos. Los cuatro presentes en Galicia son 
comunes al menos con el Duero y los del Cantábrico oriental al menos con el Ebro. Lo más destacado es la presencia en la vertiente norte del Pirineo Navarro de la lamprea de arroyo y del cavilar; este último también presente en el valle de Arán.

\section{BIBLIOGRAFIA}

BARRACHINA, P., SUYNER, C. y DOADRIO, I., (1989): «Sobre la distribución geográfica de Anaecypris hispanica (Osteichtyes, Cyprinidae)». Doñana Acta Vertebrata, 16(2): 93-95.

BLANCO, J.C., y GonZALEZ, J. L. (1992): Libro rojo de los Vertebrados de España. ICONA, Colección Técnica, MAPA, Madrid.

Coelho, M. M. (1985): «The straight mouth Portuguese Chondrostoma Agassiz, 1835. Taxonomic position and geographic distribution of Ch. Polylepis Steindachner 1965, and Ch. willkommii Steindachner 1966 with the description of a new subspecies Ch. Polylepis duriensis». Arquivos do Museu Bocage Serie A 3(2): 13-38

DOADRIO, I. (1980): «Descripción de un nuevo género y una nueva especie Iberocypris palaciosi n.gen., n.sp. (Pisces, Cyprinidae)». Doñana Acta Vertebrata, 7(1):5-17.

DOADRIO, I., (2001): Atlas y libro rojo de los peces continentales de España. Ministerio de Medio Ambiente. Org. Aut. Parques Nacionales, Madrid, 364 págs.

DOADRIO, I. y MADEIRA, M.J. (2004): «A new species of the genus Gobio Cuvier 1816 (Actinopsterugii, Cyprinidae) from the Iberian peninsula and Southwest France». Graellsia 60(1) 107-116.

DOADRIO, I. y CARMONA, J. A. (2003): «A new species of the genus Chondrostoma Agassiz, 1832 (Actinopterygii, Cyprinidae) from the Iberian Peninsula». Graellsia, 59(1): 29-36.

DOADRIO, I. y CARMONA J.A. (2006): «Phylogenetic overview of the genus Squalius (Actinopterygii, Cyprinidae) in the Iberian Peninsula, with description of two new species» Cybium 30(3):199-214

DOADRIO, I.. y CARMONA, J. A. (2009): «Pardilla oretana - Iberochondrostoma oretanum. En Enciclopedia Virtual de los Vertebrados Españoles. SALVADOR, A., y ElviRA, B. (Eds.). Museo Nac. Cienc. Nat, Madrid.

http://www.vertebradosibericos.org./

DoAdrio, I., CARmona, J.A. y Fernández-Delgado, C. (2002). Morphometric study of the Iberian Aphanius (Actinopterygii, Cyprinodontiformes), with description of a new species. Folia Zoologica: international journal of vertebrate zoology. 51(1): 67-79.

DOADRIO, I. y ElVIRA, B. (2007): «A new species of the genus Achondrostoma Robalo, Almada \& Doadrio, 2007, (Actinopterigii, ciprinidae) from western Spain». Graellsia, 63(2): 295-304

DOADRIO, I., ElVIRA, B. y BERNAT, Y., (1991): Peces continentales españoles. Inventario y clasificación de zonas fluviales. ICONA, Col. Técnica, Madrid, 221 págs. 
DoAdrio, I., KotTelat M. y SostoA, A. (2007): Squalius laietanus, a new species of cyprinid fish from north-eastern Spain and southern France (Teleostei: Cyprinidae). Ichthyol. Explor. Freshwat. 18(3):247-256.

DOADRIO,I. y PERDICES, A. (1997): « Taxonomic study of the iberian Cobittis (Osteichtyes, Cobittidae), with description of a new species». Zoological Journal of the Liennean Society: 119:51-67.

ElviRA, B. (1997): «Taxonomy of the genus Chondrostoma (Osteichthyes, Cyprinidae): an updated review». Folia Zoologica 46 (Suppl. 1): 1-14

FERRERAS CHASCO. C. y REDONDO, M. (2004): «Sobre el estado actual de conservación de la ictiofauna fluvial española endémica». En PANAREDA, J.M., Arozena, M.E., SANZ, C. y LóPEZ, N. [Eds], Estudios en Biogeografía. Libro homenaje a José Manuel Rubio Recio y Jesús García Fernández, pp. 197-202. Ed. Aster. Terrasa, Barcelona.

Ferreras Chasco, C., Redondo, M., Gonzalez Baselga, I., Abad Soria, J. y CID PEREZ, M.A. (2006): «Estado de conservación de la ictiofauna fluvial de los Montes de Toledo». III Congreso Español de Biogeografía / Espainiako III Biogeografía Biltzarra, 286-92

FERRERAS CHASCO, C. (2010): «Sobre las especies en peligro de la ictiofauna fluvial española y su problemática». En Biogeografía: una ciencia para la conservación del medio (Giménez. P., Marco. J.A., Matarredona, E., Padilla, A. y SÁncheZ, A. (Eds.) Universidad de Alicante, 211-220.

FREYHOF, J., KotTElAT, M. y NolTE, A., (2005): «Taxonomic diversity of European Cottus with description of eight new species (Teleostei: Cottidae)». Ichthyol. Explor. Freshwaters, 16 (2): 107-172

Garcia Codron, J.C., Pacheco Ibars, S. y Rasilla Alvarez, D.F. (2010): Calentamiento global y conservación del salmón atlántico». En Biogeografía: una ciencia para la conservación del medio (GIMÉNEZ. P., MARCO. J.A., Matarredona, E., Padilla, A. y Sánchez, A. eds) Universidad de Alicante, 221-233.

ICONA. (1986): Lista Roja de los vertebrados de España. Madrid.

KotTELAT, M. (1997): European freshwater fishes. Biología 52, Suppl. 5:1-27.

Kottelat, M. (2007): «Three new species of Phoxinus from Greece and southern France (Teleostei: Cyprinidae)». Ichthyological Exploration of Freshwaters, 18 (2): $145-162$. 